\title{
New Disease Reports \\ First report of a 16Srll phytoplasma (subgroup D) associated with Robinia pseudoacacia witches' broom and dieback in Iran
}

\author{
S.A. Esmaeilzadeh-Hosseini ${ }^{1}{ }^{*}$, G. Babaei $^{2}$ and S. Purmohamadi ${ }^{1}$ \\ ${ }^{1}$ Plant Protection Research Department, Yazd Agricultural and Natural Resources Research and Education Center, AREEO, \\ Yazd, Iran ; ${ }^{2}$ Plant Protection Research Department, Chaharmahal and Bakhtiari Agricultural and Natural Resources \\ Research and Education Center, AREEO, Shahrekord, Iran
}

*E-mail: esmailzadeh.phytoplasma@gmail.com

Received: 01 Nov 2019. Published: 06 Dec 2019. Keywords: black locust, Yazd

Black locust (Robinia pseudoacacia) is an important shade tree in urban green spaces in Iran. During 2017-18, R. pseudoacacia trees showing witches' broom and dieback symptoms (Fig. 1) were observed in Safayieh, Yazd province, Iran. Twenty-four symptomatic and four asymptomatic plants were surveyed for a phytoplasma and leaf samples were taken from each.

Total DNA was extracted from $0.2 \mathrm{~g}$ of fresh leaves from both diseased and symptomless plants. DNA samples were tested for the presence of phytoplasma by direct PCR primed with P1/P7 (Deng \& Hiruki, 1991; Schneider et al., 1995) and nested PCR using primers R16mF2/R16mR1 and R16F2n/R16R2 (Gundersen \& Lee, 1996). From all symptomatic plants products of the expected size (1.8. 1.4 and $1.2 \mathrm{kbp}$ ) were detected but not from symptomless plants. Restriction fragment length polymorphism (RFLP) analysis of the amplicons obtained in nested PCR with R16F2n/R16R2 primers was performed using RsaI, AluI, MseI, Hinf $\mathrm{I}$ and HaeIII restriction enzymes (Zhao et al., 2009). In all 24 positive samples, RFLP profiles were identical and related to 16SrII phytoplasmas ('Candidatus Phytoplasma aurantifolia').

R16mF2/R16mR1 amplicons from four $R$. pseudoacacia phytoplasma-infected trees were sequenced. Consensus sequences showed $100 \%$ sequence identity to each other. The sequence of an R16F2n/R16R2 amplicon (1236 bp) from a representative sample was deposited in GenBank (Accession No. MN431432). A BLAST search showed that the $R$. pseudoacacia phytoplasma shared more than $99 \%$ sequence identity with those of phytoplasma group 16 SrII. Phylogenetic analysis using the neighbour-joining method (MEGA software version 6.0) confirmed that the $R$. pseudoacacia phytoplasma clustered within the $16 \mathrm{SrII}$ phytoplasma clade and was closely related to strains in the 16SrII-D subgroup (Fig. 2). Analysis using $i$ PhyClassifier suggested that the $R$. pseudoacacia phytoplasma 16S rRNA gene RFLP pattern was identical (similarity coefficient 1.00 ) to that of a phytoplasma strain in $16 \mathrm{Sr}$ group II, subgroup D (Y10097)

A 'Ca. Phytoplasma phoenicium' strain was reported from $R$. pseudoacacia trees exhibiting yellowing symptoms in Iran (Karimzade $e$

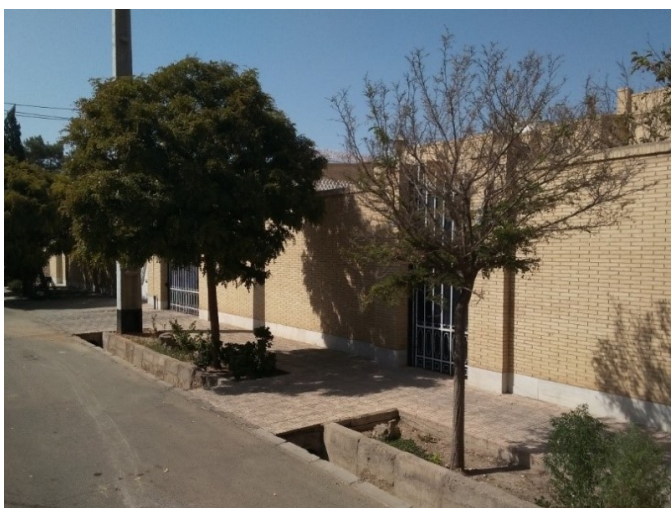

Figure 1

To cite this report: Esmaeilzadeh-Hosseini SA, Babaei G, Purmohamadi S, 2019 associated with Robinia pseudoacacia witches' broom and dieback in Iran. New Disease Reports 40, 19. http://dx.doi.org/10.5197/j.2044-0588.2019.040.019

(c)2019 The Authors

This report was published on-line at www.ndrs.org.uk where high quality versions of the figures can be found al., 2018). However, this is the first report of a phytoplasma of group 16SrII, subgroup D in $R$. pseudoacacia. The finding has a significant phytosanitary impact since this is the major phytoplasma group associated with diseases in crops and weeds in Iran and therefore poses a serious threat to other plant species growing nearby.

\section{References}

1. Deng S, Hiruki C, 1991. Amplification of 16S rRNA genes from culturable and nonculturable mollicutes. Journal of Microbiological Methods 14, 53-61.

http://dx.doi.org/10.1016/0167-7012(91)90007-D

2. Gundersen DE, Lee IM, 1996. Ultrasensitive detection of phytoplasmas by nested-PCR assays using two universal primer sets. Phytopathologia Mediterranea 35, 144-151.

3. Karimzade M, Siampour M, Zakiaghl M, Mehrvar M, 2018. Identification and characterization of a phytoplasma associated with black locust yellow disease in two provinces of Iran. Crop Protection 110, 261-268.

http://dx.doi.org/10.1016/j.cropro.2017.05.010

4. Schneider B, Seemüller E, Smart CD, Kirkpatrick BC, 1995. Phylogenetic classification of plant pathogenic mycoplasma-like organisms or phytoplasmas. In: Razin S, Tully JG eds. Molecular and Diagnostic Procedures in Mycoplasmology. New York, USA: Academic Press, 369-380.

http://dx.doi.org/10.1016/B978-012583805-4/50040-6

5. Zhao Y, Wei W, Lee I-M, Shao J, Suo X, Davis RE, 2009. Construction of an interactive online phytoplasma classification tool, $i$ PhyClassifier, and its application in analysis of the peach Xdisease phytoplasma group (16SrIII). International Journal of Systematic and Evolutionary Microbiology 59, 2582-2593. http://dx.doi.org/10.1099/ijs.0.010249-0

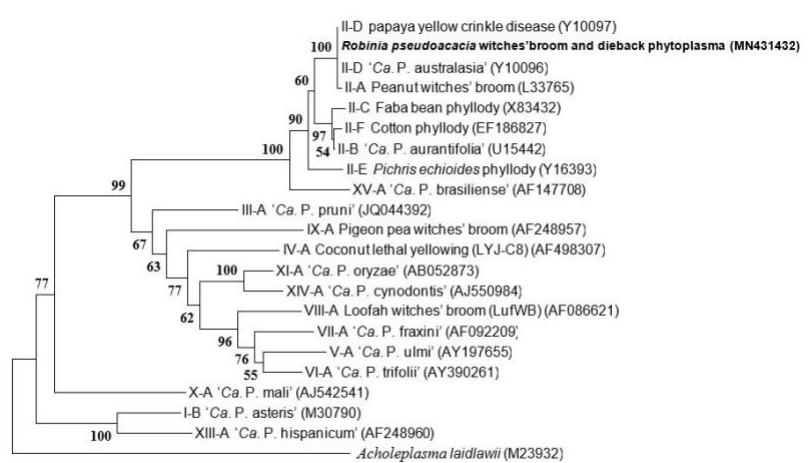

0.005 